\title{
CANNON-THURSTON MAPS DO NOT ALWAYS EXIST
}

\author{
O. BAKER ${ }^{1}$ and T. R. RILEY ${ }^{2}$ \\ ${ }^{1}$ Department of Mathematics \& Statistics, McMaster University, 1280 Main Street West, \\ Hamilton, Ontario, L8S 4K1, Canada \\ ${ }^{2}$ Department of Mathematics, 310 Malott Hall, Cornell University, Ithaca, NY 14853, USA; \\ email: tim.riley@math.cornell.edu
}

Received 25 February 2013; accepted 20 August 2013

\begin{abstract}
We construct a hyperbolic group with a hyperbolic subgroup for which inclusion does not induce a continuous map of the boundaries.
\end{abstract}

2010 Mathematics Subject Classification: 20F67

\section{Introduction}

Hyperbolic groups are the finitely generated groups whose Cayley graphs display characteristics of negative curvature. Their systematic study was initiated by Gromov in [12] and, mimicking the study of Riemannian manifolds, pays particular attention to large-scale and asymptotic geometric features such as boundaries.

One of the many equivalent definitions of the Gromov boundary $\partial G$ of an infinite hyperbolic group $G$ with word metric $d$ is as the set of equivalence classes of sequences $\left(a_{n}\right)$ in $G$ such that

$$
\left(a_{m} \cdot a_{n}\right)_{e}:=\left(d\left(a_{m}, e\right)+d\left(a_{n}, e\right)-d\left(a_{m}, a_{n}\right)\right) / 2 \rightarrow \infty
$$

as $m, n \rightarrow \infty$, where two such sequences $\left(a_{n}\right)$ and $\left(b_{n}\right)$ are equivalent when $\left(a_{m} \cdot b_{n}\right)_{e} \rightarrow \infty$ as $m, n \rightarrow \infty$. It is independent of the choice of finite generating set defining $d$ and of the choice of basepoint. See $[6,14]$ for surveys. 
When $H$ is an infinite hyperbolic subgroup of $G$, one can seek to induce a map $\partial H \rightarrow \partial G$ from the inclusion map. In [21,23] Mitra (or Mj, as he is now known) asks whether this is always well-defined, the concern being that $\partial H$ is defined via the word metric on $H$ and $\partial G$ via that on $G$, and these may differ. He cites Bonahon [5] for similar questions and Bonahon [4], Floyd [8] and Minsky [19] for related work on Kleinian groups. The question is also raised by Kapovich and Benakli [14] and appears in the problem lists [3] and [15]. When the map exists, it is known as the Cannon-Thurston map.

The Cannon-Thurston map exists for many families of examples. The most straightforward is when $H$ is quasi-convex (that is, undistorted). Cannon \& Thurston [7] gave the first distorted example: they showed that the map exists for $\pi_{1} S \hookrightarrow \pi_{1} M$ where $M$ is a closed hyperbolic 3-manifold fibering over the circle with fiber a hyperbolic surface $S$ (and, strikingly, the Cannon-Thurston map is a group-equivariant space-filling Peano curve $S^{1} \rightarrow S^{2}$ ). Mitra generalized this widely. He showed that the Cannon-Thurston map exists when $H$ is an infinite normal subgroup of a hyperbolic group $G$ [21], and he developed a theory of ending laminations for this context (inspired by [7]) to describe it [20]. He also showed that the Cannon-Thurston map exists when $H$ is one of the infinite vertex-groups or edge-groups of a finite graph of groups $G$ in which $G$ and all of the vertex-groups and edge-groups are hyperbolic, and all the defining monomorphisms from edge-groups to vertex-groups are quasi-isometric embeddings [22].

Recently, $\mathrm{Mj}$ established that Cannon-Thurston maps exist for surface Kleinian groups [25] (answering a question of Cannon \& Thurston from [7] and Question 14 from Thurston's celebrated 1982 Bulletin AMS paper [28]) and then for arbitrary Kleinian groups [24] (proving a conjecture of McMullen from [18]). Mitra's question can be viewed as asking whether the natural generalization of these results in the setting of geometric group theory holds. We answer it negatively.

THEOREM 1. There are positive words $C, C_{1}, C_{2}$ on $c_{1}, c_{2}$ and $D_{1}, D_{2}, D_{11}, D_{12}$, $D_{21}, D_{22}$ on $d_{1}, d_{2}$ such that

$$
G=\left\langle a, b, c_{1}, c_{2}, d_{1}, d_{2} \mid \begin{array}{rl}
a^{-1} b^{-1} a b=C, & b^{-1} c_{i} b=C_{i}, \\
(a b)^{-1} d_{j}(a b)=D_{j}, & c_{i}^{-1} d_{j} c_{i}=D_{i j}, \quad 1 \leq i, j \leq 2
\end{array}\right\rangle
$$

is hyperbolic, the subgroup

$$
H=\left\langle b, d_{1}, d_{2}\right\rangle
$$

is free of rank three, and there is no Cannon-Thurston map $\partial H \rightarrow \partial G$. 
A positive word is one in which inverses of generators never appear.

At the expense of complicating the construction, $G$ can be made CAT( $(-1)$, as we will outline in Remark 9.

That $H$ is free is not pertinent to the nonexistence of the Cannon-Thurston map. Theorem 1 is the starting point for a proof by Matsuda and Oguni [17] that for every nonelementary hyperbolic group there is an embedding in some other hyperbolic group for which there is no Cannon-Thurston map. Implications of Theorem 1 have also been explored by Gerasimov and Potyagailo in a recent paper [9] on convergence actions.

Given that Cannon-Thurston maps do not always exist, the question arises as to what bearing subgroup distortion has. Heavy distortion appears to be no obstacle to the map's existence: we showed in [1] that Cannon-Thurston maps exist for highly distorted free subgroups of hyperbolic hydra groups; these examples exhibit the maximum distortion known among hyperbolic subgroups of hyperbolic groups. As for small distortion, if a subgroup of a hyperbolic group is subexponentially distorted, then the subgroup is quasi-convex by [13, Proposition 2.6] and so the Cannon-Thurston map exists. The natural open question then (which Ilya Kapovich asked us) is whether there is an exponentially distorted hyperbolic subgroup of a hyperbolic group for which the Cannon-Thurston map does not exist. (An earlier version of this article claimed that the subgroup $G_{b c d} \leq G$, defined before Lemma 5, is such an example. Although $\partial G_{b c d} \rightarrow \partial G$ is not well-defined, we realized that the distortion is at least doubly exponential, so Kapovich's question remains open.) It will be apparent from our proof of Theorem 1 that the subgroup $H \leq G$ is at least doubly exponentially distorted.

\section{Proof of the theorem}

Denote the free group on a set $S$ by $F(S)$. If $S=\left\{s_{1}, \ldots, s_{n}\right\}$, write $F(S)=$ $F\left(s_{1}, \ldots, s_{n}\right)$. If $F$ is a group and $X \subseteq F$ a subset such that the natural map $F(X) \rightarrow F$ is an isomorphism, then $X$ is called a free basis for $F$ and $F$ is said to be a free group of rank $\operatorname{card}(X)$.

We begin by showing that when $C, C_{i}, D_{j}$ and $D_{i j}$ are chosen suitably, the group $G$ of Theorem 1 is hyperbolic.

A finite presentation for a group satisfies the $C^{\prime}(\lambda)$ small-cancellation condition when, after cyclically reducing all the defining relations, the set $S$ of all their cyclic permutations and those of their inverses has the property that every common prefix between two distinct $r_{1}, r_{2} \in S$ has length less than $\lambda$ times the lengths of each of $r_{1}$ and $r_{2}$ [16, p. 240]. 
Following Rips [26], we take

$$
\begin{aligned}
C & =c_{1} c_{2} c_{1} c_{2}^{2} c_{1} c_{2}^{3} \cdots c_{1} c_{2}^{r}, \\
C_{i} & =c_{1} c_{2}^{r i+1} c_{1} c_{2}^{r i+2} c_{1} c_{2}^{r+3} \cdots c_{1} c_{2}^{r i+r}, \\
D_{j} & =d_{1} d_{2}^{r j+1} d_{1} d_{2}^{r j+2} d_{1} d_{2}^{r j+3} \cdots d_{1} d_{2}^{r j+r}, \\
D_{i j} & =d_{1} d_{2}^{r(i l+j)+1} d_{1} d_{2}^{r(i l+j)+2} d_{1} d_{2}^{r(i l+j)+3} \cdots d_{1} d_{2}^{r(i l+j)+r},
\end{aligned}
$$

where $r$ is sufficiently large that the presentation for $G$ of Theorem 1 satisfies the $C^{\prime}(1 / 6)$ condition, and so $G$ is hyperbolic. (All $C^{\prime}(1 / 6)$ groups admit linear isoperimetric functions and so are hyperbolic [10].)

Next we analyze the construction of $G$ to show (via Lemmas 2 and 5(iv), (v)) that $H$ is free of rank three for such $C, C_{i}, D_{j}$ and $D_{i j}$. Specifically, we will view $G$ as being built from the free group $F\left(d_{1}, d_{2}\right)$ by $H N N$-extensions.

Suppose that $B \leq A$ are groups and $\phi: B \rightarrow A$ is any injective homomorphism (not necessarily the subgroup inclusion map). The HNN-extension $A_{* \phi}$ of $A$ with defining homomorphism $\phi$ and stable letter $t$ is the group presented by

$$
\left.A_{* \phi}:=\langle A, t| t^{-1} b t=\phi(b) \text { for all } b \in B\right\rangle,
$$

where $t$ is a new generator. (We may instead present $A_{* \phi}$ by only including the relations $t^{-1} b t=\phi(b)$ for $b$ in some particular generating set for $B$.) The groups $B$ and $\phi(B)$ are called associated subgroups of the HNN-extension.

LemMa 2 (Britton's lemma; see for example [6,16,27]). Suppose that a nonempty word $w$ on the alphabet $\{A \backslash\{e\}\} \sqcup\left\{t, t^{-1}\right\}$ contains no two consecutive letters from $A \backslash\{e\}$ and no subword $t t^{-1}$ or $t^{-1} t$. Then $w \neq 1$ in $A_{* \phi}$ unless $w$ contains a subword $t^{-1}$ bt where $b \in B$ or $t c t^{-1}$ where $c \in \phi(B)$.

In particular, the natural map $A \rightarrow A_{* \phi}$ is injective, so $A$ can be regarded as a subgroup of $A_{* \phi}$ (hence 'extension'), and $t$ generates an infinite cyclic subgroup of $A_{* \phi}$ trivially intersecting $A$.

We will need to recognize when a map between free groups is injective in order to show that it gives rise to an HNN-extension. To this end, we will want to be able to recognize free bases. Nielsen showed that a set of words represents a free basis for a subgroup of $F(X)$ when certain small-cancellation conditions are satisfied.

Proposition 3 (Nielsen; see [16, pp. 6-7]). A set $U$ of words on an alphabet $X$ represents a free basis for a subgroup of $F(X)$ if for every $v_{1}, v_{2}, v_{3} \in U^{ \pm 1}$ :

N0. $v_{1} \neq e$;

N1. $v_{1} v_{2} \neq e \Rightarrow\left|v_{1} v_{2}\right| \geq\left|v_{1}\right|,\left|v_{2}\right|$;

N2. $v_{1} v_{2} \neq e$ and $v_{2} v_{3} \neq e \Rightarrow\left|v_{1} v_{2} v_{3}\right|>\left|v_{1}\right|-\left|v_{2}\right|+\left|v_{3}\right|$. 
Corollary 4. $C, C_{1}, C_{2}$ span a rank-three free subgroup of $F\left(c_{1}, c_{2}\right)$ and $D_{1}$, $D_{2}, D_{11}, D_{12}, D_{21}, D_{22}$ span a rank-six free subgroup of $F\left(d_{1}, d_{2}\right)$.

(Indeed, N0-N2 are satisfied if $U$ satisfies the $C^{\prime}(1 / 2)$ property.)

Define

$$
\begin{aligned}
G_{c d} & :=\left\langle c_{1}, c_{2}, d_{1}, d_{2} \mid c_{i}^{-1} d_{j} c_{i}=D_{i j}, 1 \leq i, j \leq 2\right\rangle, \\
G_{b c d} & :=\left\langle G_{c d}, b \mid b^{-1} c_{i} b=C_{i}, 1 \leq i \leq 2\right\rangle .
\end{aligned}
$$

LEMMA 5. The groups defined above have the following properties.

(i) $F\left(d_{1}, d_{2}\right)$ is a subgroup of $G_{c d}$.

(ii) $F\left(c_{1}, c_{2}\right)$ is also a subgroup of $G_{c d}$ and $F\left(c_{1}, c_{2}\right) \cap F\left(d_{1}, d_{2}\right)=\{1\}$.

(iii) $G_{b c d}$ is an HNN-extension of $G_{c d}$ with stable letter $b$ and defining homomorphism $\phi: F\left(c_{1}, c_{2}\right) \rightarrow G_{b c d}$ mapping $c_{i} \mapsto C_{i}$.

(iv) $H=\left\langle b, d_{1}, d_{2}\right\rangle \leq G_{b c d}$ is free of rank three.

(v) $G$ of Theorem 1 is an HNN-extension of $G_{b c d}$ with stable letter a:

$$
G=\left\langle G_{b c d}, a \mid a^{-1} b a=b C^{-1}, a^{-1} d_{j} a=b D_{j} b^{-1}, 1 \leq j \leq 2\right\rangle .
$$

Proof. (i) By Corollary 4, the map $\phi_{1}: F\left(d_{1}, d_{2}\right) \rightarrow F\left(d_{1}, d_{2}\right)$ given by $d_{j} \mapsto D_{1 j}$ is injective. So $\phi_{1}$ defines an $\mathrm{HNN}$-extension of $F\left(d_{1}, d_{2}\right)$. Calling the stable letter $c_{1}$, this $\mathrm{HNN}$-extension has presentation

$$
G_{c_{1} d}:=\left\langle c_{1}, d_{1}, d_{2} \mid c_{1}^{-1} d_{j} c_{1}=D_{1 j}, 1 \leq j \leq 2\right\rangle .
$$

By Britton's lemma, $F\left(d_{1}, d_{2}\right) \leq G_{c_{1} d}$. Similarly, $G_{c d}$ is an HNN-extension of $G_{c_{1} d}$ with stable letter $c_{2}$ and defining homomorphism $\phi_{2}: F\left(d_{1}, d_{2}\right) \rightarrow G_{c_{1} d}$ given by $d_{j} \mapsto D_{2 j}$. Note that $\phi_{2}$ has image contained in $F\left(d_{1}, d_{2}\right) \leq G_{c_{1} d}$. Again, $\phi_{2}$ is injective by Corollary 4 . So $F\left(d_{1}, d_{2}\right) \leq G_{c_{1} d} \leq G_{c d}$ by Britton's lemma.

(ii) To show that $\left\langle c_{1}, c_{2}\right\rangle$ is a free subgroup $F\left(c_{1}, c_{2}\right)$ of $G_{c d}$ trivially intersecting $F\left(d_{1}, d_{2}\right)$, we prove the following claim. For any $n \geq 1$, any $d \in$ $F\left(d_{1}, d_{2}\right)$, and any integers $r_{0}, \ldots, r_{n+1}, s_{1}, \ldots, s_{n}$,

$$
\left(d c_{1}^{r_{0}}\right) c_{2}^{s_{1}} c_{1}^{r_{1}} c_{2}^{s_{2}} \cdots c_{1}^{r_{n}} c_{2}^{s_{n}} c_{1}^{r_{n+1}} \neq 1 \quad \text { in } G_{c d}
$$

whenever $r_{i}, s_{i} \neq 0$ for all $1 \leq i \leq n$. This, in turn, follows from Britton's lemma applied to the extension $G_{c d}$ (which has stable letter $c_{2}$ ) once we show that the $c_{1}^{r_{i}}$ are not in the associated subgroups of $G_{c d}$. As these associated subgroups are $F\left(d_{1}, d_{2}\right)$ and $F\left(D_{21}, D_{22}\right) \leq F\left(d_{1}, d_{2}\right)$, the observation that $\left\langle c_{1}\right\rangle$ is an infinite cyclic subgroup of $G_{c_{1} d}$ trivially intersecting $F\left(d_{1}, d_{2}\right)$ by Britton's lemma completes the proof.

(iii) By Corollary $4,\left\{C_{1}, C_{2}\right\}$ is a free basis of a subgroup of $F\left(c_{1}, c_{2}\right) \leq G_{b c d}$. So the defining homomorphism $\phi: F\left(c_{1}, c_{2}\right) \rightarrow G_{b c d}, c_{i} \mapsto C_{i}$ is injective. 
(iv) By Britton's lemma applied to the HNN-extension $G_{b c d}$ of $G_{c d}$, any freely reduced word $w$ on $b, d_{1}, d_{2}$ representing the identity would contain a subword $b^{ \pm 1} u b^{\mp 1}$ where $u$ is a nonempty reduced word on $d_{1}, d_{2}$ representing an element of the associated subgroup $F\left(c_{1}, c_{2}\right)$ or of the associated subgroup $\phi\left(F\left(c_{1}, c_{2}\right)\right) \leq F\left(c_{1}, c_{2}\right)$. By (ii), this is impossible. So $H:=\left\langle b, d_{1}, d_{2}\right\rangle$ is free of rank three.

(v) The given presentation for $G$ arises from that in Theorem 1 by rewriting the defining relations involving $a$. We must show that $\left\langle b C^{-1}, b D_{1} b^{-1}, b D_{2} b^{-1}\right\rangle \leq$ $G_{b c d}$ is free of rank three. It suffices to show the same of the conjugate subgroup $\left\langle b^{-1} C, D_{1}, D_{2}\right\rangle \leq G_{b c d}$. We do this by proving that if $i_{1}, \ldots i_{r-1} \neq 0$ and $W_{1}, \ldots, W_{r}$ are nontrivial elements of the rank-two free group $F\left(D_{1}, D_{2}\right) \leq$ $F\left(d_{1}, d_{2}\right) \leq G_{b c d}$, then

$$
w:=\left(b^{-1} C\right)^{i_{0}} W_{1}\left(b^{-1} C\right)^{i_{1}} \cdots W_{r}\left(b^{-1} C\right)^{i_{r}}
$$

does not represent the identity in $G_{b c d}$. This is achieved by writing $w$ in such a way that Britton's lemma applies.

The relations $b^{-1} c_{i} b=C_{i}$ imply that $\left(b^{-1} C\right)^{i_{k}} \in\left\langle c_{1}, c_{2}\right\rangle b^{-i_{k}}\left\langle c_{1}, c_{2}\right\rangle$, so

$$
w \in\left\langle c_{1}, c_{2}\right\rangle b^{-i_{0}}\left\langle c_{1}, c_{2}\right\rangle W_{1}\left\langle c_{1}, c_{2}\right\rangle b^{-i_{1}}\left\langle c_{1}, c_{2}\right\rangle \cdots W_{r}\left\langle c_{1}, c_{2}\right\rangle b^{-i_{r}}\left\langle c_{1}, c_{2}\right\rangle .
$$

If $b^{ \pm 1}$ does not appear in $w$, then $r=1, i_{0}=i_{1}=0$, and $w=W_{1}$ does not represent the identity in $G_{b c d}$. So we may assume that $b$ appears. To apply Britton's lemma, we must show that $w$ has no subword $b^{ \pm 1} Y b^{\mp 1}$ where $Y$ is a word on $c_{1}, c_{2}, d_{1}, d_{2}$ representing an element of $F\left(c_{1}, c_{2}\right)$. This is so because $F\left(c_{1}, c_{2}\right) \cap F\left(d_{1}, d_{2}\right)=\{1\}$ by (ii) and $W_{k} \in F\left(d_{1}, d_{2}\right) \leq G_{b c d}$ does not represent the identity.

We will use the following lemma of Mitra to show the absence of a Cannon-Thurston map $\partial H \rightarrow \partial G$. We give our own account of this lemma in [1].

LemMa 6 (Mitra [21,22]). Suppose that $H$ is a hyperbolic subgroup of a hyperbolic group $G$ and $X_{H}$ and $X_{G}$ are their Cayley graphs with respect to finite generating sets where that for $H$ is a subset of that for $G$. (So $X_{H}$ is a subgraph of $X_{G}$.) Let $M(N)$ be the infimal number such that if $\lambda$ is a geodesic in $X_{H}$ outside the ball of radius $N$ about $e$ in $X_{H}$, then every geodesic in $X_{G}$ connecting the end-points of $\lambda$ lies outside the ball of radius $M(N)$ about $e$ in $X_{H}$. The Cannon-Thurston map $\partial H \rightarrow \partial G$ exists if and only if $M(N) \rightarrow \infty$ as $N \rightarrow \infty$.

We will apply this to $G$ and $H$ of Theorem 1, using the generating sets $a, b, c_{1}, c_{2}, d_{1}, d_{2}$ and $b, d_{1}, d_{2}$, respectively.

The next lemma identifies some geodesics in Cayley graphs of smallcancellation groups. We learnt it from Ilya Kapovich and Hamish Short. It can 
be extracted from Strebel's appendix to [11] as we will explain. For a finite presentation $\langle A \mid R\rangle$, a word $w$ on $A$ is Dehn-reduced if every subword $\alpha$ of $w$ that is a prefix of a cyclic conjugate of some $\rho \in R^{ \pm 1}$ satisfies $|\alpha| \leq|\rho| / 2$, and is strongly Dehn-reduced if $|\alpha| \leq|\rho| / 6$.

Lemma 7. If $\langle A \mid R\rangle$ is a $C^{\prime}(1 / 6)$-presentation, then every strongly Dehnreduced word on $A$ is geodesic. (Indeed, it is the unique geodesic word and also the unique Dehn-reduced word for the group element that it represents.)

Proof. Suppose that $u$ and $v$ are freely reduced words which represent the same group element, and $u$ is strongly Dehn-reduced and $v$ is geodesic. In his proof of Proposition 39(i) in his appendix to [11], Strebel explains that there is a van Kampen diagram $\Delta$ for $u v^{-1}$ whose two-dimensional portions are ladder-like disc-diagrams. (See the figure within Theorem 35.)

Suppose that there is a 2-cell in $\Delta$ and that $\rho$ is the defining relation that one reads around its boundary. That 2-cell's boundary cycle is assembled from four paths: two run along the boundaries of adjacent 2-cells and have lengths less than $|\rho| / 6$ (by the $C^{\prime}(1 / 6)$ condition); one runs along $u$ and has length at most $|\rho| / 6$ by the strongly Dehn-reduced condition; but then the final path, which runs along $v$, has length more than $|\rho| / 2$ contrary to $v$ being a geodesic word. (Indeed, if we only required $v$ to be Dehn-reduced we would get the same contradiction.) So $\Delta$ has no 2-cells and $u=v$ as words.

Proof of Theorem 1. Recall that

$$
G=\left\langle a, b, c_{1}, c_{2}, d_{1}, d_{2} \mid \begin{array}{rl}
a^{-1} b^{-1} a b=C, & b^{-1} c_{i} b=C_{i}, \\
(a b)^{-1} d_{j}(a b)=D_{j}, & c_{i}^{-1} d_{j} c_{i}=D_{i j}, 1 \leq i, j \leq 2
\end{array}\right\rangle
$$

where

$$
\begin{aligned}
C & =c_{1} c_{2} c_{1} c_{2}^{2} c_{1} c_{2}^{3} \cdots c_{1} c_{2}^{r}, \\
C_{i} & =c_{1} c_{2}^{r i+1} c_{1} c_{2}^{r i+2} c_{1} c_{2}^{r i+3} \cdots c_{1} c_{2}^{r i+r}, \\
D_{j} & =d_{1} d_{2}^{r j+1} d_{1} d_{2}^{r j+2} d_{1} d_{2}^{r j+3} \cdots d_{1} d_{2}^{r j+r}, \\
D_{i j} & =d_{1} d_{2}^{r(i l+j)+1} d_{1} d_{2}^{r(i l+j)+2} d_{1} d_{2}^{r(i l+j)+3} \cdots d_{1} d_{2}^{r(i l+j)+r} .
\end{aligned}
$$

We must show that for sufficiently large $r, G$ is hyperbolic, $H=\left\langle b, d_{1}, d_{2}\right\rangle$ is free of rank three, and there is no Cannon-Thurston map $\partial H \rightarrow \partial G$.

As we observed at the start of this section, $G$ can be made hyperbolic by choosing $r$ large enough to make $G$ satisfy $C^{\prime}(1 / 6)$. Britton's lemma and Lemma 5(iv), (v) together show that $H$ is a rank-three free subgroup for the same $r$. We may assume that $r>17$. It remains to show that the Cannon-Thurston map does not exist. 


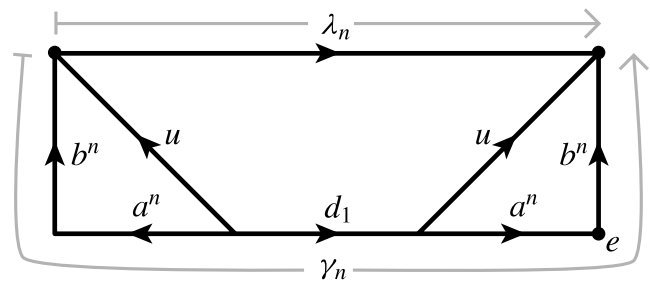

Figure 1. Paths in the Cayley graph of $G$ illustrating our proof of Theorem 1.

The longest subword of $b^{-n} a^{-n} d_{1} a^{n} b^{n}$ that is a prefix of a cyclic conjugate of a defining relation or the inverse of a defining relation is $a^{-1} d_{1} a$. Since $r>17$, the length of $a^{-1} d_{1} a$ is a small fraction (less than 1/6) of the length of the shortest of the relators. So $b^{-n} a^{-n} d_{1} a^{n} b^{n}$ is strongly Dehn-reduced. So, by Lemma 7 , the path $\gamma_{n}$ that it labels, passing through the identity $e$ as shown in Figure 1, is geodesic in the Cayley graph of $G$.

We now wish to express $b^{-n} a^{-n} d_{1} a^{n} b^{n}$ as a word in $d_{1}, d_{2}$. To begin, we prove by induction on $n$ that

$$
a b^{n}=b^{n-1} a b \phi(C) \cdots \phi^{n-2}(C) \phi^{n-1}(C)
$$

in $G$, where $\phi: F\left(c_{1}, c_{2}\right) \rightarrow G_{b c d}, c_{i} \mapsto C_{i}$ is the defining homomorphism of the HNN-extension $G_{b c d}$ with stable letter $b$-see Lemma 5 (iii). The base case $n=1$ is the equation $a b=a b$. The induction step follows from the relation $a^{-1} b^{-1} a b=C$ (which rearranges to $a b=b a C$ ):

$$
\begin{aligned}
a b^{n+1}=(a b) b^{n}=(b a C) b^{n} & =b\left(a b^{n}\right)\left(b^{-n} C b^{n}\right) \\
& =b\left(a b^{n}\right) \phi^{n}(C) \\
& =b\left(b^{n-1} a b \phi(C) \cdots \phi^{n-2}(C) \phi^{n-1}(C)\right) \phi^{n}(C),
\end{aligned}
$$

where the last equality uses the induction hypothesis. Left-multiplying (2.1) by $a^{n-1}$ yields

$$
a^{n} b^{n}=\left(a^{n-1} b^{n-1}\right) a b \phi(C) \cdots \phi^{n-2}(C) \phi^{n-1}(C)
$$

Another induction then shows that $a^{n} b^{n}$ can be written as a positive word $u$ in the alphabet $\left\{a b, c_{1}, c_{2}\right\}$. So $b^{-n} a^{-n} d_{1} a^{n} b^{n}=u^{-1} d_{1} u$ in $G$, which equals a positive word on $d_{1}, d_{2}$ since $(a b)^{-1} d_{j}(a b)=D_{j}$ and $c_{i}^{-1} d_{j} c_{i}=D_{i j}$ in $G$.

So the end-points of $\gamma_{n}$ are in $H$, and the geodesic $\lambda_{n}$ joining them in the Cayley graph of $H$ (which is a tree) is labeled by a word on $d_{1}, d_{2}$. The distance (along the path labeled $b^{n}$ ) from $e$ to $\lambda_{n}$ in $H$ is $n$. 
As the distance from $\gamma_{n}$ to $e$ in the Cayley graph of $G$ is zero and the distance from $\lambda_{n}$ to $e$ in the Cayley graph of $H$ is $n$, there is no Cannon-Thurston map $\partial H \rightarrow \partial G$ by Lemma 6 .

\section{Remarks}

REMARK 8. The inclusion $H \hookrightarrow G$ factors through $G_{b c d}$, which is also hyperbolic as its presentation is also $C^{\prime}(1 / 6)$. So Theorem 1 implies the absence of at least one Cannon-Thurston map $\partial H \rightarrow \partial G_{b c d}$ or $\partial G_{b c d} \rightarrow \partial G$. In fact, more elaborate versions of the argument given above establish that both fail to exist. As an $\mathrm{HNN}$-extension is an example of a graph of groups, the latter example also shows that the quasi-isometric embedding hypothesis in Mitra's theorem from [22] is necessary.

REMARK 9. With a similar construction, one can obtain a CAT $(-1)$ group $G$ with a free subgroup $H$ with no Cannon-Thurston map. Wise's modification in [29] of the Rips construction [26] is used in [2] to construct CAT(-1) groups. Each relator is realized on the boundary of the unions of $n=5$ congruent right-angled regular hyperbolic pentagons, arranged as row houses atop a geodesic segment. Each edge of the boundary corresponds to a generator. The vertices of the boundary are either right angles or straight angles, but the base geodesic gives $n-1$ consecutive straight angles, bounding a segment of length $n-2$. Wise shows that the Gromov link condition is satisfied when this straight segment is a freely reduced word and when the length- $(2 n+4)$ remainder of the boundary is obtained from the Wise word:

$$
c_{1}\left(c_{1} c_{2} c_{1} c_{3} \cdots c_{1} c_{r}\right) c_{2}\left(c_{2} c_{3} c_{2} c_{4} \cdots c_{2} c_{r}\right) c_{3}\left(c_{3} c_{4} \cdots c_{3} c_{r}\right) \cdots c_{r-1}\left(c_{r-1} c_{r}\right) c_{r}
$$

by chopping it into consecutive length $2 n+4$ segments (one for each defining relator). The argument works just as well for any $n$, so we take $n=7$ and fit the $(a b)^{-1} d_{j}(a b), a^{-1} b^{-1} a b, b^{-1} c_{i} b$, and $c_{i}^{-1} d_{j} c_{i}$ portions of our relators along the straight segment. We form one Wise word of $c \mathrm{~s}$ and one of $d \mathrm{~s}$. To get sufficiently many length-18 subwords of the Wise words, we increase the number of $c_{i}$ and $d_{j}$ in the generating set for $G$. Then $H=\left\langle b, d_{1}, d_{2}, \ldots\right\rangle$ is a free subgroup of the CAT $(-1)$ group $G$ by the same argument as before.

REMARK 10. $H$ has infinite height in $G$. That is, for all $n$, there exist $g_{1}, \ldots, g_{n} \in G$ such that $\bigcap_{i=1}^{n} g_{i}{ }^{-1} H g_{i}$ is infinite and $H g_{i} \neq H g_{j}$ for all $i \neq j$. Specifically, take $g_{i}=c_{1}^{i}$. Then, if $\phi_{1}: F\left(d_{1}, d_{2}\right) \rightarrow F\left(d_{1}, d_{2}\right)$ is the map $d_{j} \mapsto D_{1 j}$ for $j=1,2$, then $\phi_{1}^{n}\left(F\left(d_{1}, d_{2}\right)\right)$ is an infinite subgroup inside $g_{i}^{-1} H g_{i}$ for $1 \leq$ $i \leq n$, and $H g_{i} \neq H g_{j}$ for all $i \neq j$ since $c_{1}^{k} \in H$ only for $k=0$ by Lemma 5 . Likewise, $G_{b c d}$ has infinite height in $G$ : instead of taking $g_{i}=c_{1}^{i}$, take $g_{i}=(a b)^{i}$ 
and apply the same argument as above. So our examples do not resolve the question attributed to Swarup in [23]: if $H$ is a finitely presented subgroup of a hyperbolic group $G$ and $H$ has finite height in $G$, is $H$ quasiconvex in $G$ ?

\section{Acknowledgements}

We thank Ilya Kapovich and Hamish Short for comments and especially for Lemma 7, which replaces a more ad hoc argument in an earlier version of this article. We are also grateful to an anonymous referee for a careful reading, improvements to our exposition, and insights on the background to Mitra's problem. The first author held a Postdoctoral Fellowship at McMaster University at the time the research on this paper was conducted. The second author gratefully acknowledges partial support from NSF Grant DMS-1101651 and from Simons Foundation Collaboration Grant 208567.

\section{References}

[1] O. Baker and T. R. Riley, Cannon-Thurston maps for hyperbolic hydra. arXiv:1209.0815, (2012).

[2] J. Barnard, N. Brady and P. Dani, 'Super-exponential distortion of subgroups of CAT(-1) groups', Algebr. Geom. Topol. 7 (2007), 301-308.

[3] M. Bestvina, Questions in geometric group theory. http://www.math.utah.edu/ bestvina/.

[4] F. Bonahon, 'Bouts des variétés hyperboliques de dimension 3', Ann. of Math. (2) 124(1) (1986), 71-158.

[5] F. Bonahon, 'Geodesic currents on negatively curved groups', in Arboreal Group Theory (Berkeley, CA, 1988), Math. Sci. Res. Inst. Publ., 19 (Springer, New York, 1991), 143-168.

[6] M. R. Bridson and A. Haefliger, Metric Spaces of Non-Positive Curvature, Grundlehren der mathematischen Wissenschaften, 319 (Springer, 1999).

[7] J. W. Cannon and W. P. Thurston, 'Group invariant Peano curves', Geom. Topol. 11 (2007), 1315-1355.

[8] W. J. Floyd, 'Group completions and limit sets of Kleinian groups', Invent. Math. 57(3) (1980), 205-218.

[9] V. Gerasimov and L. Potyagailo, Similar relatively hyperbolic actions of a group. arXiv: 1305.6649, (2013).

[10] S. M. Gersten, 'Introduction to hyperbolic and automatic groups', in Summer School in Group Theory, Banff, 1996, CRM Proc. Lecture Notes, 17 (American Mathematical Society, Providence, RI, 1999), 45-70.

[11] E. Ghys and P. de la Harpe (eds.) Sur les Groups Hyperbolic d'après Mikhael Gromov, Progress in Mathematics, 83 (Birkhäuser, 1990).

[12] M. Gromov, 'Hyperbolic groups', in Essays in Group Theory, MSRI Publications, 8 (ed. S. M. Gersten) (Springer, 1987), 75-263.

[13] I. Kapovich, 'The combination theorem and quasiconvexity', Internat. J. Algebra Comput. 11(2) (2001), 185-216. 
[14] I. Kapovich and N. Benakli, 'Boundaries of hyperbolic groups', in Combinatorial and Geometric Group Theory (New York, 2000/Hoboken, NJ, 2001), Contemporary Mathematics, 296 (American Mathematical Society, Providence, RI, 2002), 39-93.

[15] M. Kapovich, Problems on boundaries of groups and Kleinian groups, collected at a 2005 American Institute of Mathematics workshop. http://www.math.ucdavis.edu/ kapovich/ EPR/problems.pdf.

[16] R. C. Lyndon and P. E. Schupp, Combinatorial Group Theory, Classics in Mathematics (Springer, Berlin, 2001), Reprint of the 1977 edition.

[17] Y. Matsuda and S. Oguni, On Cannon-Thurston maps for relatively hyperbolic groups. arXiv:1206.5868, (2012).

[18] C. T. McMullen, 'Local connectivity, Kleinian groups and geodesics on the blowup of the torus', Invent. Math. 146(1) (2001), 35-91.

[19] Y. N. N. Minsky, 'On rigidity, limit sets, and end invariants of hyperbolic 3-manifolds', J. Amer. Math. Soc. 7(3) (1994), 539-588.

[20] M. Mitra, 'Ending laminations for hyperbolic group extensions', Geom. Funct. Anal. 7(2) (1997), 379-402.

[21] M. Mitra, 'Cannon-Thurston maps for hyperbolic group extensions', Topology 37(3) (1998), $527-538$

[22] M. Mitra, 'Cannon-Thurston maps for trees of hyperbolic metric spaces', J. Differential Geom. 48 (1998), 135-164.

[23] M. Mitra, 'Coarse extrinsic geometry: a survey', in The Epstein Birthday Schrift, Geom. Topol. Monogr., 1 (Geometry \& Topology Publications, Coventry, 1998), 341-364.

[24] M. Mj, Cannon-Thurston maps for Kleinian groups. arXiv:1002.0996, (2010).

[25] M. Mj, 'Cannon-Thurston maps for surface groups', Annals of Math. 179 (2014), 1-80.

[26] E. Rips, 'Subgroups of small cancellation groups', Bull. Lond. Math. Soc. 14(1) (1982), 45-47.

[27] J. Stillwell, Classical Topology and Combinatorial Group Theory, 2nd edn. Graduate Texts in Mathematics (Springer, 1993).

[28] W. P. Thurston, 'Three-dimensional manifolds, Kleinian groups and hyperbolic geometry', Bull. Amer. Math. Soc. (N.S.) 6(3) (1982), 357-381.

[29] D. T. Wise, 'Incoherent negatively curved groups', Proc. Amer. Math. Soc. 126(4) (1998), 957-964. 